\title{
Simulation Research of Evolutionary Game to Bank and Technological SME under the Pledge Financing \\ Mode
}

\author{
Huafeng Chen ${ }^{1,2,3}$, Mu Zhang ${ }^{1}$ \\ ${ }^{1}$ School of Finance, Guizhou University of Finance and Economics, Guiyang Guizhou 550025, China \\ ${ }^{2}$ Guizhou Institute for Urban Economics and Development, Guizhou University of Finance and Economics, Guiyang \\ Guizhou 550025, China \\ ${ }^{3}$ Guizhou Institution for Technology Innovation \& Entrepreneurship Investment, Guizhou University of Finance and
}

Economics, Guiyang Guizhou 550025, China

\author{
质押贷款下银行与科技型中小企业演化博交的仿真研 \\ 究 \\ 陈华丰 ${ }^{1,2,3}$, 张目 ${ }^{1}$ \\ 1贵州财经大学金融学院, 贵州 550025, 中国 \\ 2 贵州财经大学贵州城镇经济与发展研究院, 贵州 550025 , 中国 \\ 3 贵州财经大学贵州科技创新创业投资研究院, 贵州 550025 , 中国
}

\begin{abstract}
In this paper, Matlab is used to simulation research based on evolutionary game mode of technological SME and bank under the pledge of intellectual Property financing. The evolutionary game results are analyzed to 3 different evolutionary strategies. Game strategies are not converged to a stable value simultaneously, there are the leader and later. Lacking of a stable point, the simulation can also judge the approximate location of the midpoint. Also in some cases, the stable value is depended on the initial value, and the order of size of the stable value is not entirely dependent on the order of size of the initial value.
\end{abstract}

Keywords: simulation research; evolutionary game; technological SME; The pledge of intellectual property

\section{摘要}

本文主要运用 Matlab 对基于知识产权质押银行与科 技型中小企业的演化博弯模型进行了仿真模拟。分析 了 3 种不同演化策略下模型演化博弯的结果。发现博
弯双方的策略并不是同时收玫到稳定值, 有领先者也 有 “后知后觉” 者。在没有稳定点情况下, 也可以通 过仿真判断中点的大概位置。还发现在某些情况下, 稳定值取决于起始博亦值, 且稳定值的大小排序并不 是完全取决于起始值的大小排序。

关键词: 仿真模拟; 演化博亦; 科技型中小企业; 知 识产权质押

1. 引言

随着科学技术的迅猛发展和全球经济一体化进程的 加快, 科技型中小企业在我国经济发展中扮演的角色 愈发重要, 作为提高综合国力和国家竞争优势的重要 源泉, 科技型中小企业的发展关系整个国家经济的未 来[1]。但是科技型中小企业具有高风险、规模小、 有形资产少、无形资产多和信用级别低等特点, 导致 了它融资十分困难。2006年10月, 交通银行北京分行 发放全国范围内首例中小企业知识产权质押贷款, 开 启了知识产权质押贷款商业化推广的序幕 [2]。知识 产权质押贷款比较适合科技型中小企业的特点, 但是 知识产权质押贷款也有一系列的局限。知识产权的价 


\section{Risk Analysis and Crisis Response in Big Data Era (RAC-16)}

值评估难、风险管理难、变现难，以及其适用的法律 法规和配套措施还不够完善。银行作为主要放贷机 构, 面对科技型中小企业和知识产权质押贷款的一系 列特点, 以及放贷过程中的信息不对称和道德风险。 这使得银行产生 “惜贷” 现象，使知识产权质押贷款 的实施艰难, 科技型中小企业融资困难。

本文基于演化博弯理论, 构建了银行与科技型中 小企业的演化博亦模型，运用Matlab对模型进行了仿 真模拟, 并对仿真结果进行分析并给出建议。旨在从 风险管理的角度探讨银行与科技型中小企业的演化 博弯过程，降低知识产权质押贷款的风险，减少双方 博弯过程中不必要的损失, 促成银行与科技型中小企 业长期稳定合作。

Greenwald和Stieglitz(1986)研究发现中小企业难 以获得贷款是因为信息的不完全和契约的不完备而 造成的, 建议通过国家垄断力量解决信息和契约的问 题来改善中小企业融资困境[3]。彭湘杰(2007)分析了 知识产权质押融资的可行性, 并建议运用有效的登记 制度、电子公示、集合财产担保、执行程序简化和多 渠道融资的方式来推动知识产权质押融资的发展 [4]。宋伟, 胡海洋(2009)认为知识产权质押融资过高 的风险（包括知识产权质押的法律风险、知识产权质 押的经济风险、知识产权变现风险) 和由高风险产生 的高交易成本（包括支付给交易第三方的成本、金融 机构风险定价产生高利率成本) 是制约知识产权质押 融资发展的主要瓶颈[5]。李云飞, 周宗放（2011） 建立了符合我国实际情况的风险投资项目风险评价 指标体系, 针对单个风险投资项目的风险等级确定问 题和多个风险投资项目的风险排序问题, 分别构建模 型予以解决最后给出算例说明了这两个模型的具体 应用[6]。曾江洪, 肖涛, 王庄志(2013)将银行与中小 企业间的动态博亦过程视为一个 “模仿学习” 的渐进 系统, 构建中小企业抵押贷款及互助担保贷款的演化 博亦模型对比分析两种贷款模式的演化路径及其相 应条件，探讨如何缓解中小企业融资难问题[7]。杨 扬，陈敬良(2014)运用演化博亦方法，建立了我国高 新技术企业知识产权质押融资行为的演化博弯模型。 研究发现, 高新技术企业知识产权质押融资行为选择 演化系统既可以收玫到帕累托最优均衡状态, 也可以 收敛帕累托劣均衡状态 [8]。

综上所述, 国内外关于知识产权质押贷款和银行 与中小企业演化博亦的研究成果丰富, 但银行与科技 型中小企业演化博亦的仿真模拟的成果较少。本文在 文献[9]所建立的模型基础上, 运用Matlab仿真分析了 3种不同演化策略下模型演化博弯结果。最后, 对博
栾双方的策略选择和贷款制度的制定给出一些建议。

\section{2. 演化博弯模型建立}

演化博亦理论的形成和发展大致经历三个阶段：首 先, 生物学家从博亦论中得到启示, 运用博亦论建构 各种生物竞争演化模型, 包括动物竞争、性别分配以 及植物的成长和发展等: 接着, 生物学家根据生物演 化的自身规律, 对传统博亦论进行改造, 包括将传统 博亦论中支付函数转化为生物适应度函数、引入突变 机制将传统的纳什均衡精炼为演化稳定均衡以及引 入选择机制建构复制者动态模型。随后，鉴于演化博 弯对传统博弯的拓展, 经济学家又反过来借鉴生物学 家的思想, 将演化博亦运用到经济学中, 这又进一步 推动演化博竕的发展 $[10]$ 。

在博弯过程中, 银行与科技型中小企业都是为了 追求自身利益最大化。但是自身利益不仅取决于自身 策略的选择也取决于对方策略的选择。在每次博亦 后, 银企双方会对自身和当前情况作出总体评价, 学 习同类中收益较高者的行为, 调整自己的策略。

\section{1. 模型假设及银企支付矩阵}

在该模型中, 供贷者为银行(博亦方 1 ), 借贷者为科 技型中小企业(博亦方 2$)$ 。

假设科技型中小企业因投资一项目需要借贷 $B$, 投资成功的收益率为 $\alpha$, 投资失败的收益为 0 , 银行 只知道企业创新项目的平均成功率为 $\lambda$ 。银行的放贷 利率为 $r$, 居民到银行的存款利率为 $k$ 。由于科技型 中小企业拥有大量先进的技术但实体资产较少, 因此 知识产权质押的融资方式更适合科技型中小企业。故 假设科技型中小企业知识产权价值为 $C_{0}$, 质押率为 $l$ 。随着知识和技术的更新, 质押的知识产权有贬 值的风险，假设质押期间的贬值率为 $q$ 。质押的知识 产权的价值评估委托给第三企业, 费用由银行承担为 $C$ 。为了防范企业贷后的道德风险及违约行为, 银行 需要建立起核查机制和奖惩机制。当科技型中小企业 投资成功并按时还款时, 将受到银行的奖励 $S$ 。当科 技型中小企业投资成功但出于私利谎称失败, 出现违 约骗贷现象。经核查确认将受到银行的惩罚 $R$ 。骗贷 现象出现概况为 $p$, 核查的成本为 $V L$, 且 $V L<p R$ 。

根据上述模型假设。相应的银行和科技型中小企 业的博亦支付矩阵如表1。

\section{2. 演化博弯中的平衡点}

根据上述假设和支付矩阵, 我们假设 $Y$ 为银行对 借贷企业进行核查的概率, $1-Y$ 为银行对企业不进 
Risk Analysis and Crisis Response in Big Data Era (RAC-16)

表 1 银行与科技型中小企业的支付矩阵

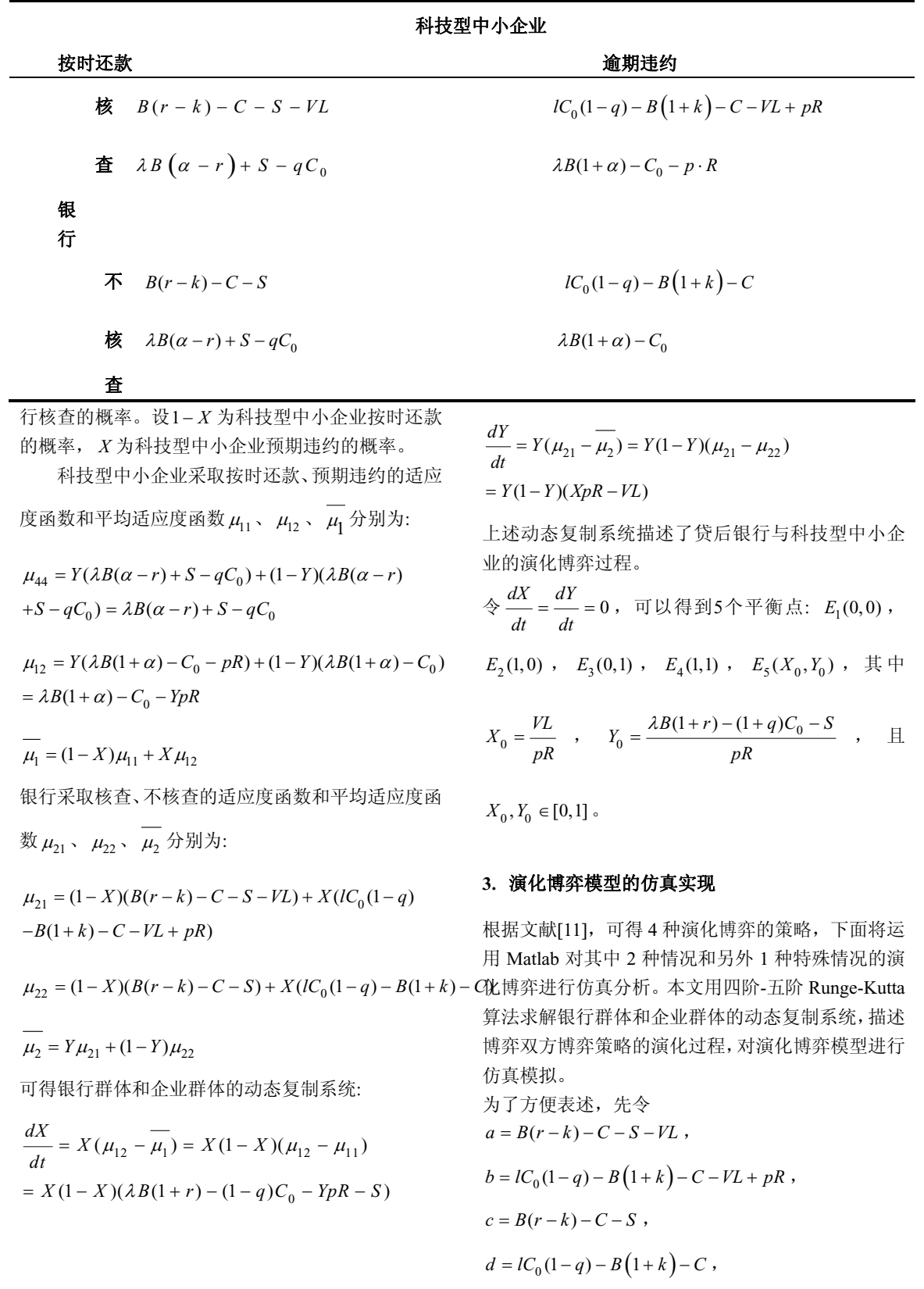


Risk Analysis and Crisis Response in Big Data Era (RAC-16)

$$
\begin{array}{ll}
e=\lambda B(\alpha-r)+S-q C_{0}, & g=\lambda B(\alpha-r)+S-q C_{0}, \\
f=\lambda B(1+\alpha)-C_{0}-p \cdot R, & h=\lambda B(1+\alpha)-C_{0}
\end{array}
$$

表 2 参数组合

\begin{tabular}{|c|c|c|c|c|c|c|c|c|c|c|c|c|c|}
\hline 变量 & $B$ & $\alpha$ & $\lambda$ & $r$ & $k$ & $C_{0}$ & $l$ & $q$ & $C$ & $S$ & $R$ & $p$ & $V L$ \\
\hline 参数 & 200 & $50 \%$ & $80 \%$ & $4.9 \%$ & $2.75 \%$ & 100 & $70 \%$ & $3 \%$ & 5 & 30 & 100 & $25 \%$ & 20 \\
\hline
\end{tabular}

(1) 演化策略 1

当 $a<c, b>d, e<f, g<h$ 时, 有 4 个平衡点,

$E_{4}(1,1)$ 为稳定点。演化博弯的稳定点策略为银行选

择 “核查”, 科技型中小企业选择 “预期违约”。设置 参数如表 2 , 图 1 中左图设置的起始博栾点为: $X=0$, $0.1,0.2,0.3,0.4,0.5,0.6,0.7,0.8,0.9,1: \mathrm{Y}=0.1$; 博弯周期 $\mathrm{T}$ 为 0.5 , 图 1 右图设置的起始博弯点为: $\mathrm{X}=0.1 ＼textrm{Y}=0,0.1,0.2,0.3,0.4,0.5,0.6,0.7,0.8$, $0.9,1$; 博亦周期 $\mathrm{T}$ 为 3 , 进行仿真模拟, 得图 1 。 所得仿真结果与理论结果一致。如图分析, X 的收玫 速度较快, 在 $0.2 \mathrm{~T}$ 时所有博弯中 $\mathrm{X}$ 策略已经趋向于 1。且 $X$ 得起始博弯值设置的越大, 它趋向于稳定的 速度就越快。 $\mathrm{Y}$ 的收玫速度相对较慢, 大约在 $2 \mathrm{~T}$ 时 所有博弯中 $Y$ 策略已经趋向于 1 。且 $Y$ 的起始博弯 值设置的越大, 它趋向于稳定的速度就越快。在博栾 刚开始时, $Y$ 策略的值的变化为先减小再增大, 最后 收玫于 1 。在此种参数设置条件下, 科技型中小企业 选择 “预期违约” 所获得的收益更大, 所以企业的策 略很明确, 选择 “预期违约” 的概率不断增大, 最终 $\mathrm{X}$ 收玫于 1 。但是银行选择 “核查” 时, 需要支付核 查成本 VL, 所以在博弯开始时银行的策略选择倾向 于 “不核查”, 故图 2 中 Y 策略的值出现先减小的情 况。但在博弯过程中银行改变了策略, 逐渐倾向 “核 查”, 最终 Y 收玫于 1 。我们还可以发现, 博弯双方 的策略选择并不是同时收玫的, 有领先者也有 “后知 后觉” 者。当一方的策略以及演化到稳定, 另一方仍

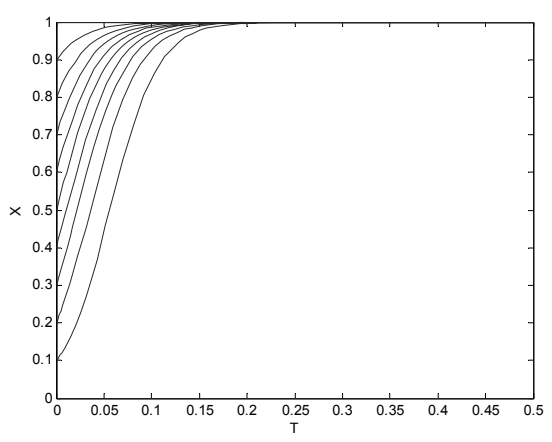
在继续演化。

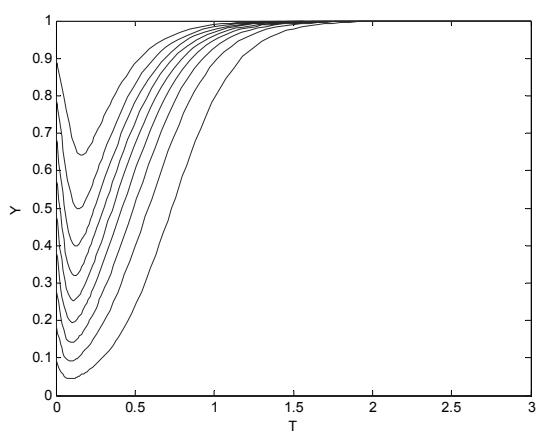

图 1 演化策略 1 中 $\mathrm{X}$ 与 $\mathrm{Y}$ 单独的演化过程

表 3 参数组合 2

\begin{tabular}{|c|c|c|c|c|c|c|c|c|c|c|c|c|c|}
\hline 变量 & $B$ & $\alpha$ & $\lambda$ & $r$ & $k$ & $C_{0}$ & $l$ & $q$ & $C$ & $S$ & $R$ & $p$ & $V L$ \\
\hline 参数 & 200 & $50 \%$ & $80 \%$ & $4.9 \%$ & $2.75 \%$ & 100 & $70 \%$ & $3 \%$ & 5 & 30 & 200 & $25 \%$ & 20 \\
\hline
\end{tabular}

(2) 演化博弯 2

当 $a<c, b>d, e>f, g<h$ 时, 有 5 个平衡点

$X_{0}=\frac{V L}{p R} \quad, \quad Y_{0}=\frac{\lambda B(1+r)-(1+q) C_{0}-S}{p R}$, 且

但没有稳定点, $E_{5}\left(X_{0}, Y_{0}\right)$ 为中心。其中

$X_{0}, Y_{0} \in[0,1]$ 。设置参数如表 3 , 根据参数设置, 可 
Risk Analysis and Crisis Response in Big Data Era (RAC-16)

计算出理论数值 $X_{0}=0.4, Y_{0}=0.8168$ 。图 2 设置的 起始博亦点为: $X=0,0.1,0.2,0.3,0.4,0.5,0.6$, $0.7,0.8,0.9,1: \mathrm{Y}=0,0.1,0.2,0.3,0.4,0.5$, $0.6,0.7,0.8,0.9,1$; 博亦周期 $\mathrm{T}$ 为 5 , 一共有 121 组博亦组合。图 3 设置的起始博亦点为: $X=0.1$; $\mathrm{Y}=0.1$; 博亦周期 $\mathrm{T}$ 为 200 。图 7 设置的起始博亦点 为: $X=0.1 ; Y=0.1$; 博亦周期 $T$ 为 200 。进行仿

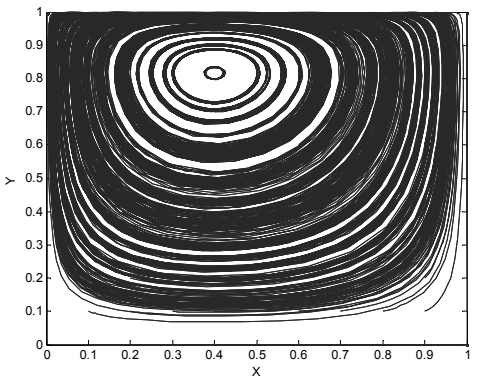

图 2 演化博亦 2 中 $\mathrm{X}$ 与 $\mathrm{Y}$ 共同的演化过程

真模拟, 得图 3 。从仿真中计算得出 $X_{0} \in[0.375,0.425], Y_{0} \in[0.797,0.835]$, 所得仿真 结果的数值与理论数值比较接近。如图分析, $\mathrm{X}$ 与 $\mathrm{Y}$ 共同的博亦演化过程呈环形, 没有稳定点。 $\mathrm{Y}$ 取值的 变化会影响 $\mathrm{X}$ 取值的变化, $\mathrm{X}$ 取值的变化又会影响 $\mathrm{Y}$ 取值的变化。为了自身利益的最大化, 科技型中小企 业的策略选择会根据银行的策略选择而不断调整, 银 行的策略选择也会根据科技型中小企业的策略选择 而不断调整。 $\mathrm{X}$ 取值的最大值先逐渐减小, 最终趋向 于稳定; $X$ 取值的最小值先逐渐增大, 最终趋向于稳 定。 $\mathrm{Y}$ 取值的最大值一直为 $1 ; \mathrm{Y}$ 取值的最小值先增 大最终趋向于稳定。 $\mathrm{X}$ 与 $\mathrm{Y}$ 某数值被选取的频繁程 度可从图 3 中颜色的深浅能大概了解。图 3 中左图颜

色较深的大概区域是 $X_{0} \in[0.1,0.5]$, 图 3 中右图颜色

较深的大概区域是 $Y_{0} \in[0.7,1]$ 。因此也能从图 3 中大 概的判断出中心的位置。

\section{（3）演化策略 3}

当 $a<c, b>d, e>f, g=h$ 时, 在通常理 论分析中很少讨论这种情况, 我们运用仿真进行分 析。仿真模拟后发现没有稳定点。设置参数如表 4, 图 4 左图设置的起始博亦点为: $\mathrm{X}=0,0.1,0.2,0.3$,
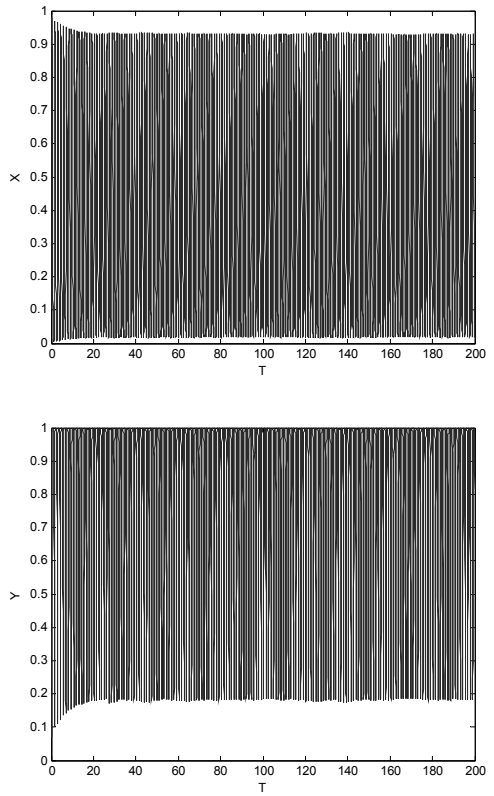

图 3 演化博亦 2 中 $\mathrm{X}$ 与 $\mathrm{Y}$ 单独的演化过程

$0.4,0.5,0.6,0.7,0.8,0.9,1: \mathrm{Y}=0.1$; 博弯周期 $\mathrm{T}$ 为 5 , 图 4 右图设置的起始博弯点为: $X=0.1 ; Y=0$, $0.1,0.2,0.3,0.4,0.5,0.6,0.7,0.8,0.9,1$; 博 弯周期 $\mathrm{T}$ 为 1 , 进行仿真模拟, 得图 4。如图分析, 因为设置的起始博弯点不同, $\mathrm{X}$ 的稳定值也不同, 且 稳定值的大小排序并不是完全取决于起始值的大小 排序。图 4 左图中, $X$ 起始值为 0.9 的最终稳定值要 小于起始值为 0.7 的最终稳定值。但 $\mathrm{Y}$ 的博亦演化 过程没有出现这样 “越界” 的情况。因此, 我们可以 得到结论: 在某些情况下, 稳定值取决于起始博弯值, 且稳定值的大小排序并不是完全取决于起始值的大 小排序。

\section{4. 研究结论及建议}

本文基于银行与科技型中小企业演化博弯模型。首先 仿真模拟了 3 种不同演化策略下的演化结果, 并对这 3 种演化博弯情况进行研究分析。得到以下结论。

（1）博亦双方的策略并不是同时收玫到稳定值, 有 领先者也有 “后知后觉”者。当一方的策略以及演化到 稳定, 另一方仍在继续演化。

（2）在没有稳定点情况下，也可以通过仿真判断中 
Risk Analysis and Crisis Response in Big Data Era (RAC-16)

表 4 参数组合 3

\begin{tabular}{|c|c|c|c|c|c|c|c|c|c|c|c|c|c|}
\hline 变量 & $B$ & $\alpha$ & $\lambda$ & $r$ & $k$ & $C_{0}$ & $l$ & $q$ & $C$ & $S$ & $R$ & $p$ & $V L$ \\
\hline 参数 & 200 & $50 \%$ & $80 \%$ & $4.9 \%$ & $2.75 \%$ & 100 & $70 \%$ & $3 \%$ & 5 & 70.84 & 100 & $25 \%$ & 20 \\
\hline
\end{tabular}
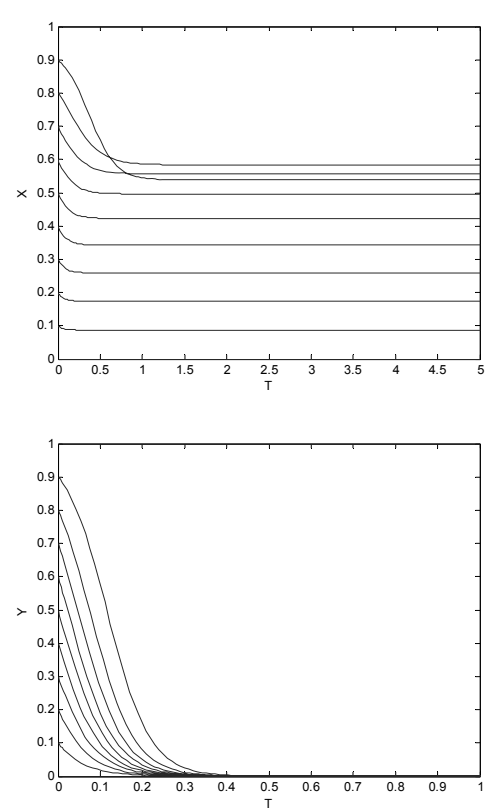

图 4 演化博弯 3 中 $\mathrm{X}$ 与 $\mathrm{Y}$ 单独的演化过程

点的大概位置。

（3）某些情况下, 稳定值取决于起始博亦值, 且稳 定值的大小排序并不是完全取决于起始值的大小排 序。

根据研究, 建议银行和科技型中小企业应谨慎选 择首次博弯的策略。根据演化策略 3 的结果首次博弯 值的选择可能会影响到最终的稳定结果。

\section{Acknowledgements}

This paper was one of the achievements of National Natural Science Foundation of China of regional project 《Research on Loan Risk Compensation Funds for Technological SME's Effect Mechanism of the Credit Rationing》 (71263011).

\section{致谢}

本文为国家自然科学基金地区项目《贷款风险补
偿资金对科技型中小企业信贷配给的影响机理研究》 (71263011)的阶段性成果之一。

\section{5. 参考文献}

[1] 苑泽明. 知识产权融资的风险、估价与对策. 第 一版东北财经大学出版社, 2010 .

[2] 袁吉伟. 科技型中小企业知识产权质押贷款探 析. 财会研究, 2008(5): 69-70.

[3] B.C. Greenwald, J. E Stieglitz.. Externalities in economies with imperfect information and incomplete markets. Quarterly Journal of Economics. 1986, (101): 229-264.

[4] 彭湘杰. 知识产权质押融资理论发展、实践与借 鉴中国金融．中国金融，2007(5): 23-24.

[5] 宋伟, 胡海洋. 知识产权质押贷款风险分散机制 研究. 知识产权, 2009(4):73-77.

[6] Y.F. Li, Z.F. Zhou. Research on model for evaluating risks of venture capital projects. Journal of Risk Analysis and Crisis Response, 2011, 1(2):142-148.

[7] 曾江洪, 肖涛, 王庄志. 基于演化博亦的中小企 业贷款模式对比研究. 数学的实践与认识, 2013, 42(17):60-68.

[8] 杨扬, 陈敬良. 我国高新技术企业知识产权质押 融资机制的演化博亦分析. 工业技术经济, 2014(7):43-48.

[9] 陈华丰, 张目, 张红梅. 知识产权质押融资模式 下银行与科技型中小企业的演化博亦分析. 新 常态下西部金融创新与风险控制论坛暨第四届 中国西部风险分析与风险管理学术研讨会, 成 都, 2015.

[10] 黄凯南. 演化博亦与演化经济学. 经济研究, 2009(1):132-145.

[11] 张林，肖诗顺. 中小企业融资难的博亦分析. 商 业经济, 2011(6):95-96. 